\title{
Cerebral correlates of disturbed executive function and memory in survivors of severe closed head injury: a SPECT study
}

\author{
G Goldenberg, W Oder, J Spatt, I Podreka
}

\begin{abstract}
Thirty six patients in the chronic stage after severe closed head injury were examined with tests of executive function, memory, intelligence, and functional capacities in daily living. Correlations were sought between test results and Tc$99 \mathrm{~m}-$ HMPAO uptake of frontal, temporal, and thalamic regions assessed by SPECT. Neither the number of significant correlation coefficients between memory tests and regional uptake nor that between temporal uptake and tests exceeded chance. For the remaining tests, correlations to thalamic regions were stronger than those to the frontal regions, and those to right brain regions stronger than those to homologous left brain regions. Relationships of thalamic isotope uptake to neuropsychological performance may reflect the impact of diffuse brain damage and particularly of diffuse axonal injury on mental capacities.
\end{abstract}

Impairment of memory and lack of executive control over cognitive function and behaviour constitute major obstacles to the successful reintegration of survivors of severe head injury (SHI). ${ }^{1-6}$ Clinical neuropsychology associates memory impairment with mesial temporal lobe or diencephalic lesions, and dysexecutive syndrome with frontal lobe damage. Left-sided lesions affect predominantly the manipulation and storage of verbal, and right-sided lesions that of non-verbal material. The frontal and temporal lobes are frequent sites of contusion in SHI. ${ }^{6-10}$ MRI studies found executive function and memory impairment to be associated with frontal and temporal lobe lesions in the acute stage of moderately severe head trauma, ${ }^{11}$ but failed to establish convincing relationships between neuropsychology and either the intrahemispheric localisation or the laterality of lesions in the chronic stages of severe head injury. ${ }^{12-14}$ By contrast they found a relationship between the depth of lesions and the general level of neuropsychological performance in both acute and chronic stages of severe head injury. ${ }^{91213}$ Since deep lesions occur predominantly in association with diffuse axonal injury (DAI), ${ }^{8-1015}$ these results seem to indicate that long-term psychological outcome after SHI is mainly determined by the presence and severity of DAI but depends much less on the presence and localisation of contusions. The prominence of dysexecutive syndrome and memory impairment may then be due to the fact that these functions are particularly vulnerable to the multiple interruptions of intracerebral connections caused by DAI.

Brain damage can result not only from immediate impact injury but also from complications in the later course of SHI. Raised intracranial pressure can injure the basal and mesial temporal lobes either by pressure necro$\operatorname{sis}^{810}$ or by ischaemia as a result of compression of the posterior artery stem. The hippocampal formation is particularly vulnerable to diffuse degeneration of cortical neurons in cerebral hypoxia. ${ }^{8}$ Finally, haematomas and ischaemic infarctions in watershed areas of vascular supply may affect temporal or frontal regions although they do not show a specific predilection for these areas of the brain.

The heterogeneity of pathogenetic mechanisms of brain injury is possibly a reason for the failure to find consistent relationships between the localisation of lesions and the pattern of neuropsychological impairment in SHI. Only some of the pathological alterations are accompanied by structural changes visible on MRI or CT. Functional brain imaging by means of PET or SPECT can detect dysfunction of regions which look structurally intact, and thus promises a more comprehensive analysis of the substrates of memory impairment and dysexecutive syndrome in SHI. ${ }^{1617}$

The purpose of this study was to compare the distribution of regional cerebral blood flow (rCBF) as measured by Tc-99m-HMPAOSPECT to dysexecutive syndrome, memory impairment, and functional capacities in daily living in survivors of severe head injury.

\section{Patients}

Thirty six patients ( 5 female, 31 male) who had been admitted to our department for early rehabilitation after severe head injury were readmitted for a comprehensive clinical, neuropsychological, and radiological re-examination. The mean time elapsed since the trauma was 39.3 months (range 7 to 66 ). The mean initial Glasgow Coma Scale was 6.6 (range 3-14). The minimum duration of posttraumatic amnesia was 5 days. Posttraumatic amnesia was not longer than 1 week in 3 patients, between 1 week and 1 month in 2 patients, between 1 and 2 months in 14 patients, and longer than 2 months in 17 patients.

There were two groups of controls. A group of 29 controls ( 9 female, 20 male) of similar age and educational level was examined with 
the neuropsychological test-battery in the same way as the patients. These controls were not investigated by SPECT. Control data for SPECT were taken from a study on $\mathrm{rCBF}$ in visual imagery ${ }^{18}$ in which volunteers had been examined in a resting state. The SPECT control group was comparable to patients with respect to gender-distribution and age, but the mean educational level was higher.

\section{NEUROPSYCHOLOGICAL ASSESSMENT}

The assessments were classified to test executive function, memory, or general intelligence, and whether they were more sensitive to disturbance of visuospatial or verbal functions. To reduce data, compound scores were calculated for all tests which yielded more than one result.

\section{Executive Function}

Association Learning: ${ }^{19}$ The patient was shown consecutively nine cards with coloured geometrical figures on their frontside. Three cards had a black dot on the back. The patient was asked to memorise which cards bore dots. The frontside of each card was shown for approximately 5 seconds, then the card was turned and the presence or absence of the dot was demonstrated. After the initial presentation the cards were shuffled, the patient was shown one card after the other again and was asked to say whether there was a dot on its back or not. Feedback was given by turning the card. This was repeated 5 times, the cards being shuffled between each run. The score was twice the number of correct responses minus the number of false alarms (chance score $=0$ ). Repeated false alarms to the same card were scored as perseverations.

In a second run a new set of cards were presented which were identical on the front side but had the dots on different cards. The patient was informed about this change, and the second run was administered and scored as the first one. For statistical evaluation a compound score was calculated which excluded both, a low score on the second run and a drop of the score from the first to the second run. (see footnote 1, page 368).

Card Sorting. The patient was given eight cards with coloured figures which could be sorted into two equally sized groups according to six possible categories. The patient was asked to group them repeatedly using different categories. The score was the number of categories found.

Tower of London: ${ }^{1920}$ The patient had to move three coloured beads from a starting configuration on three sticks of unequal length to a target configuration without moving more than one bead at a time. There were 12 problems of increasing difficulty and 1 minute was allowed for each of them. The score was the number of problems solved within the time limit with less than 15 moves.

Word Fluency: The patient was given 3 minutes to say as many words starting with " $S$ " as possible. They were advised to avoid repetitions and proper names. The score was the number of words produced.
Design Fluency: ${ }^{21}$ The patient was given a sheet partitioned in rectangles. Five symmetrically arranged dots were printed in each rectangle. The patient was asked to produce as many figures as possible within 3 minutes by connecting dots within each square, but to avoid repetitions of figures.

A perseveration score was calculated from the number of perseverations in association learning, word fluency and design fluency. The number of rule breaks was summed up across card-sorting (unequal group sizes, illogical groupings), Tower of London (attempts to move more than one bead at a time), word fluency (production of proper names), and design fluency (disregard of dots).

Verbal Working Memory: The tests of working memory capacity employed a concurrent span paradigm. ${ }^{22}$ First, word span was assessed. The patient was presented a sequence of printed two- to three-syllables low-imagery nouns. They were asked to read aloud each word and finally to repeat the whole sequence in the correct order. Two trials each were given with increasing lengths of sequences until the patient failed with both trials of a length. The length of the last successfully repeated sequence was scored as single span.

The patient was then presented with sequences of meaningful sentences of 10 to 15 words which ended on a two to three syllables lowimagery noun. They were asked to read aloud the whole sentences but to memorise only the last word of them, and to repeat the last words in the correct order after the end of a sequence. Scoring was the same as with the single span.

A compound score evaluated the length of the span with concurrent task, and the drop from single to concurrent task performance. (see footnote 2, page 368).

Visuospatial Working Memory: $:^{23}$ For assessment of a spatial span, the patient was presented with a sequence of sheets with a large square printed on each of them. Within the large square there was a small square which contained a Chinese letter. The location of the small square was different for each sheet of a series. The patient was asked to memorise the locations of the letters. Immediately after the end of the sequence, the patient was shown the large square covered by an empty $5 \times 5$ grid and asked to point to the locations that corresponded to the memorised locations. Each of the memorised locations fit exactly to one of the small squares of the response grid. Reproduction of the order of presentation was not required.

The patient was again presented sequences of large squares, each of which contained one small square with a Chinese letter, but outside the large square there was an additional Chinese letter which was either identical to the one within or differed by one feature. The patient was asked to compare both Chinese letters, to say whether they were identical or not, and to memorise the locations of the letters within the square. The location of the additional letter did not vary. After a sequence, the response grid was presented and the patient was asked to recall the locations as in the single span 
procedure.

The scoring system for visuospatial working memory was the same as that for the verbal working memory.

Current theories of working memory ${ }^{24}$ assume that it requires a collaboration of modality specific slave systems with a supramodal central executive. Introduction of concurrent tasks enhances demands on the central executive. The compound scores emphasised the drop in performance from single to concurrent span performance and should thus be particularly sensitive to a disturbance of the central executive component of working memory. Scores on the working memory tests are interpreted as measures of executive function.

\section{Secondary Memory}

Logical Memory: ${ }^{25}$ Both stories of the Wechsler Memory Scale were read to the patient and free recall was tested immediately after presentation of each story and again half an hour later. The compound score was the mean number of ideas recalled at both occasions.

Rey Figure Memory: ${ }^{25}$ The Rey-Osterrieth complex figure was given for copy, immediate recall, and delayed recall after half an hour. Scoring was done as suggested by Osterrieth. ${ }^{25}$ The compound score was the mean of both recall scores. The compound score was not corrected for the completeness of the copy to make it comparable to Logical Memory which does not control for incomplete or erroneous apprehension of the memoranda.

Recognition Memory for Words: The patients were presented with a sequence of 25 onesyllable high frequency nouns each printed on one page of a booklet. They were asked to read aloud each word and to memorise them. They were then shown 25 listed words intermingled with 25 high-frequency one-syllable nouns which had not been contained in the list, and were asked if the words had been presented before. This was repeated after 30 minutes with different distractors. The score was the number of correct positive minus the number of false positive responses, averaged across immediate and delayed recognition.

Recognition Memory for Faces: The patients were presented with 25 consecutive photographs of unfamiliar male faces. They were asked to comment on whether they found them pleasant or not and to memorise them. Then they were presented one after the other the same faces intermingled with 25 male faces not shown before and were asked to say for each face whether it had already been presented. This was repeated after 30 minutes with different distractors. Scoring was done as with recognition memory for words.

\section{Intelligence:}

Vocabulary: The MWT-B ${ }^{26}$ was administered. This is a multiple-choice lexical decision test, where 37 words of decreasing frequency have to be selected among 4 phonemically similar non-words. The score is the number of correct choices.

Ravens's coloured progressive matrices ${ }^{27}$ were administered and scored in the usual way.
Verbal and visuospatial specialisation of tests: Of the tests of executive functions, fluency and working memory each consisted of a verbal and of a visuospatial version. For secondary memory, logical memory and word recognition tested verbal, and Rey figure memory and face recognition visuospatial function. Vocabulary and progressive matrices differ in several ways apart from being differentially sensitive to disturbances of verbal and visuospatial functions, therefore differences between them will not be related to laterality effects.

Testing was performed in two sessions on separate days. Within each session, the order of tests was fixed to avoid interference effects between different memory tasks, but the order of the two sessions was varied randomly.

Functional capacities were rated according to a scale ${ }^{28}$ which consists of two to three point rank scales evaluating engagement in occupation, capacity to handle financial affairs, capacity to manage domestic responsibilities, capacity to perform activities of daily living, and the need for facilities providing care.

\section{SPECT}

For SPECT, patients were placed in a supine position with their eyes closed and the ears unplugged. SPECT was performed after injection of approximately $20 \mathrm{mCi}$ Tc-99mHMPAO by means of a dual head rotating gamma camera equipped with high resolution collimators which yield a spatial resolution of $12 \mathrm{~mm}$ FWHM in the axial plane. ${ }^{29}$ After filtering, reconstruction and attenuation correction, 4 consecutive axial slices of $21 \mathrm{~mm}$ thickness were displayed and regions of interest were drawn by hand according to anatomical templates (figure). Regions were drawn in the hemisphere that showed less lesions and were then mirrored to the other hemisphere. Indices of regional isotope uptake were calculated by dividing the count rate of a region through the mean count rates of all regions taken together.

The conditions of data acquisition and the mode of evaluation was the same for the SPECT control group, with the exception that the controls wore blindfolds in addition to keeping their eyes closed, that they were explicitly advised to relax and "think of nothing", and that regions of interest were drawn by hand in both hemispheres. ${ }^{18}$

HMPAO uptake is largely proportional to regional cerebral blood flow, but the relationship is a curvilinear one which may become irregular under conditions of impaired bloodbrain barrier. ${ }^{30}{ }^{31}$ We will discuss the results of HMPAO-SPECT as reflecting regional cerebral blood flow and designate indices of regional isotope uptake as regional flow indices. Reduction of regional flow indices can result not only from reduced blood flow in normal tissue volume but also from normal blood flow in reduced tissue volume and therefore reflects both structural and functional tissue damage.

Neuropsychological testing, assessment of functional capacities and evaluation of SPECT 

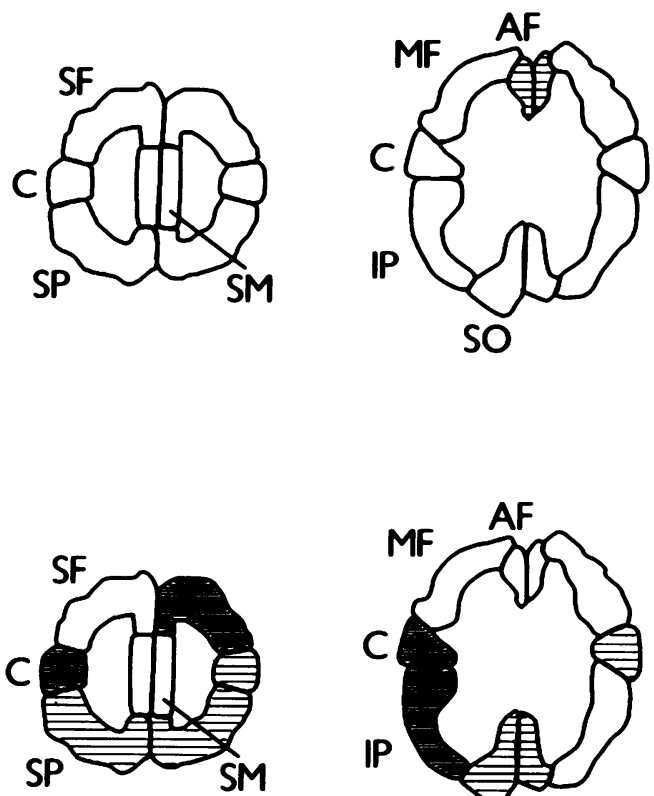

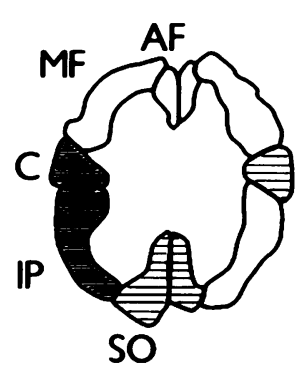

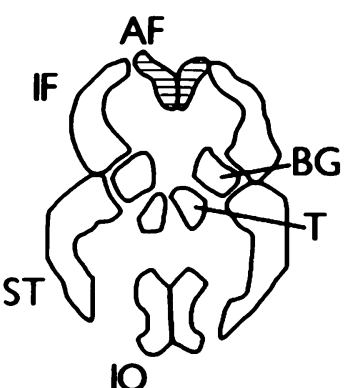
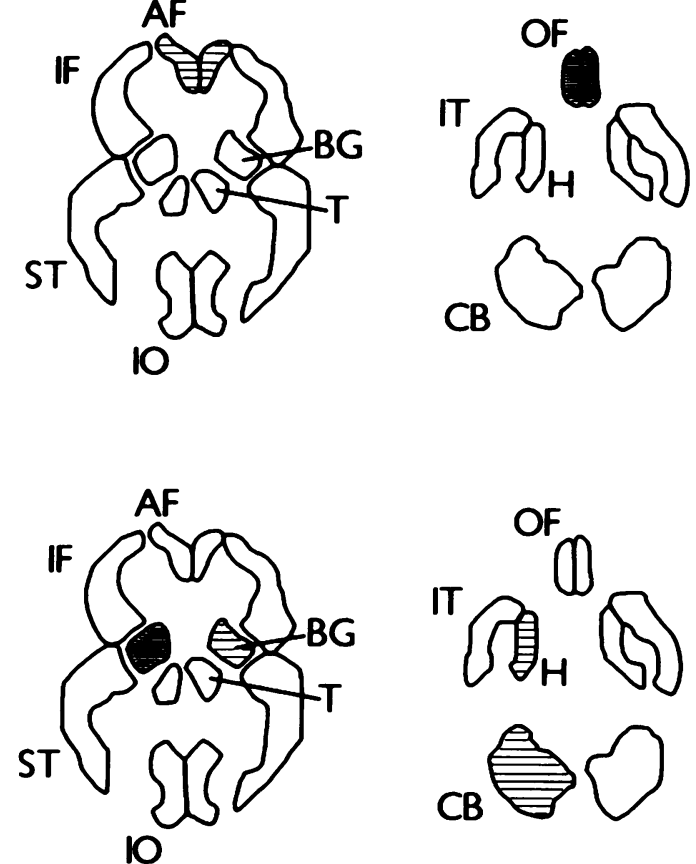

Figure Regions of interest from a normal control's SPECT study and significant differences between flow rates of patients and controls. Top: flow rates of patients lower than of controls; bottom: flow rates of patients higher than of controls; hatched regions: $p<0.05$ as single comparison; dark regions: $p<0.05$ after Bonferroni-Holms correction for

Sultiple tests. orbitofrontal; C: central; SP: superior parietal; IP: inferior parietal; SO: superior occipital; IO: inferior occipital; ST: superior temporal; IT: inferior temporal; H: hippocampus; BG: basal ganglia; T: thalamus; CB: cerebellum. The left side of the slices corresponds to the left side of the brain.

were performed independently each by one of the authors.

\section{Results}

Performance on neuropsychological tests

Test results of patients and controls are shown in table 1. Patients performed worse than controls on all tests. With Bonferroni-Holms ${ }^{32}$ correction for multiple comparisons, the differences were significant at a global probability of 0.05 for all results except the number of perseverations, the scores on design fluency and both intelligence tests.

\section{SPECT}

The figure shows significant differences of regional flow indices between patients and controls. As a group, patients had lower flow indices bilaterally in orbitofrontal and anterofrontal regions, but higher flow values in both basal ganglia and central regions, the left hippocampus region, the left inferior parietal region, the right superior frontal region, and in both superior occipital and superior parietal regions. After Bonferroni-Holms ${ }^{32}$ correction for multiple comparisons, the differences remained significant for both orbitofrontal, the right superior frontal, the left central, the left inferior parietal, and the left basal ganglia region. Thalamic flow indices were lower in patients than in controls, but the difference was not significant $(P=0.13$ for the left, and 0.32 for the right side).

Table 1 Demographic data and neuropsychological test results of patients and controls

\begin{tabular}{|c|c|c|c|c|c|}
\hline & $\begin{array}{l}36 \text { Patients } \\
\text { Mean }\end{array}$ & $(S D)$ & $\begin{array}{l}29 \text { Controls } \\
\text { Mean }\end{array}$ & $(S D)$ & $P$ (t test) \\
\hline $\begin{array}{l}\text { Age } \\
\text { Years of Education } \\
\text { Association Learning } \\
\text { Card Sorting } \\
\text { Tower of London } \\
\text { Word Fluency } \\
\text { Design Fluency } \\
\text { Perseverations } \\
\text { Rule Breaks } \\
\text { Verbal Working Memory } \\
\text { Spatial Working Memory } \\
\text { Logical Memory } \\
\text { Rey Figure Memory } \\
\text { Word Recognition } \\
\text { Face Recognition } \\
\text { Vocabulary } \\
\text { Progressive Matrices }\end{array}$ & $\begin{aligned} 30 \cdot 2 \\
10 \cdot 0 \\
17 \cdot 1 \\
3 \cdot 3 \\
10 \cdot 3 \\
20 \cdot 7 \\
25 \cdot 0 \\
8 \cdot 9 \\
2 \cdot 9 \\
2 \cdot 1 \\
1 \cdot 9 \\
7 \cdot 9 \\
17 \cdot 4 \\
12 \cdot 0 \\
13 \cdot 1 \\
24 \cdot 5 \\
31 \cdot 7\end{aligned}$ & $\begin{aligned}(10 \cdot 5) \\
(3 \cdot 5) \\
(11 \cdot 9) \\
(1 \cdot 3) \\
(1 \cdot 8) \\
(7 \cdot 5) \\
(11 \cdot 1) \\
(8 \cdot 3) \\
(2 \cdot 7) \\
(1 \cdot 3) \\
(1 \cdot 8) \\
(3 \cdot 0) \\
(7 \cdot 3) \\
(3 \cdot 8) \\
(5 \cdot 6) \\
(5 \cdot 3) \\
(4 \cdot 4)\end{aligned}$ & $\begin{aligned} 29 \cdot 4 \\
10 \cdot 7 \\
26 \cdot 6 \\
4 \cdot 4 \\
11 \cdot 7 \\
27 \cdot 2 \\
31 \cdot 0 \\
4 \cdot 8 \\
0 \cdot 7 \\
3 \cdot 3 \\
3 \cdot 2 \\
10 \cdot 6 \\
25 \cdot 0 \\
15 \cdot 0 \\
17 \cdot 1 \\
27 \cdot 9 \\
33 \cdot 0\end{aligned}$ & $\begin{array}{l}(11 \cdot 4) \\
(2 \cdot 3) \\
(5 \cdot 7) \\
(1 \cdot 4) \\
(0.6) \\
(7 \cdot 5) \\
(8 \cdot 5) \\
(4 \cdot 8) \\
(0.8) \\
(1 \cdot 1) \\
(1 \cdot 6) \\
(2 \cdot 3) \\
(5 \cdot 1) \\
(2 \cdot 7) \\
(2.3) \\
(5.5) \\
(2.3)\end{array}$ & $\begin{aligned}> & 0.1 \\
> & 0.1 \\
< & 0.0005^{\star} \\
& 0.004^{\star} \\
< & 0.0005^{\star} \\
& 0.001^{\star} \\
& 0.018 \\
& 0.017 \\
< & 0.0005^{\star} \\
< & 0.0005^{\star} \\
& 0.003^{\star} \\
< & 0.0005^{\star} \\
< & 0.0005^{\star} \\
& 0.001^{\star} \\
< & 0.0005^{\star} \\
& 0.016 \\
> & 0.1\end{aligned}$ \\
\hline
\end{tabular}

^Significant after Bonferroni-Holms correction to a global alpha probability of 0.05 . 
Table 2 Spearman rank correlations between regional flow rates and test performance

\begin{tabular}{|c|c|c|c|c|c|c|}
\hline & $\begin{array}{l}\text { Left } \\
\text { Frontal }\end{array}$ & $\begin{array}{l}\text { Right } \\
\text { Frontal }\end{array}$ & $\begin{array}{l}\text { Lefi } \\
\text { Temporal }\end{array}$ & $\begin{array}{l}\text { Right } \\
\text { Temporal }\end{array}$ & $\begin{array}{l}\text { Left } \\
\text { Thalamus }\end{array}$ & $\begin{array}{l}\text { Right } \\
\text { Thalamus }\end{array}$ \\
\hline Association Learning & 0.22 & $0 \cdot 18$ & -0.03 & $-0 \cdot 11$ & $0 \cdot 17$ & $0 \cdot 07$ \\
\hline Card Sorting & $0 \cdot 32^{\star}$ & $0.47^{\star \star}$ & $0 \cdot 21$ & 0.26 & ${ }^{\star} 0.46^{\star}$ & $0 \cdot 53^{\star \star \star}$ \\
\hline Tower of London & $-0 \cdot 17$ & -0.20 & -0.25 & -0.27 & $0 \cdot 14$ & $0.31^{\star}$ \\
\hline Word Fluency & $0 \cdot 18$ & 0.09 & $0 \cdot 12$ & $-0 \cdot 14$ & $0.29^{\star}$ & $0 \cdot 27$ \\
\hline Design Fluency & $0 \cdot 14$ & $0 \cdot 27$ & 0.00 & -0.04 & $0.45^{\star \star}$ & $0.55^{\star \star \star}$ \\
\hline Perseverations & $0 \cdot 15$ & $0 \cdot 17$ & -0.08 & $-0 \cdot 24$ & $0 \cdot 16$ & $0 \cdot 11$ \\
\hline Rule Breaks & $0 \cdot 33^{\star}$ & $0 \cdot 47^{\star \star}$ & -0.05 & -0.03 & 0.25 & $0 \cdot 46^{\star \star}$ \\
\hline Verbal Working Memory & $0 \cdot 14$ & $0 \cdot 32^{\star}$ & $-0 \cdot 12$ & 0.03 & -0.05 & $0 \cdot 10$ \\
\hline Spatial Working Memory & 0.09 & $0 \cdot 29^{\star}$ & -0.07 & $0 \cdot 19$ & 0.24 & $0 \cdot 39 \star \star$ \\
\hline Logical Memory & -0.12 & 0.02 & 0.01 & -0.28 & 0.28 & $0 \cdot 24$ \\
\hline Rey Figure Memory & $-0 \cdot 14$ & 0.00 & -0.27 & 0.08 & $0 \cdot 18$ & $0.37^{\star}$ \\
\hline Word Recognition & -0.13 & $0 \cdot 07$ & 0.00 & -0.02 & -0.02 & 0.01 \\
\hline Face Recognition & -0.02 & 0.22 & $-0 \cdot 29^{\star}$ & -0.07 & -0.05 & $0 \cdot 28$ \\
\hline Vocabulary & $0 \cdot 25$ & $0.41^{\star \star}$ & $0 \cdot 45^{\star \star}$ & $0.43^{\star \star}$ & $0 \cdot 56^{\star \star \star}$ & $0.50^{\star \star}$ \\
\hline Progressive Matrices & $0 \cdot 30^{\star}$ & $0 \cdot 39^{\star \star}$ & 0.05 & $0 \cdot 11$ & $0 \cdot 32^{\star}$ & $0.51^{\star \star \star}$ \\
\hline Functional Capacities & $0 \cdot 36^{\star}$ & $0 \cdot 46^{\star \star}$ & -0.04 & 0.08 & $0 \cdot 17$ & $0 \cdot 40^{\star \star}$ \\
\hline
\end{tabular}

Signs are arranged that a positive correlation associates better performance with higher flow rates. ${ }^{\star}: \mathrm{p}<0.05$; ${ }^{\star \star}: \mathrm{p}<0.01$; $\star \star \star: \mathrm{p}<0.001$ which is equivalent to a global $\mathrm{p}<0.05$ after Bonferroni-Holms correction.

Since flow indices reflect the distribution but not the absolute size of rCBF, local elevations need not necessarily indicate true hyperaemia. They may result from a relative preservation of local flow in the presence of diffuse hypoperfusion. In this case, the pattern of differences would correspond to predominant damage of polar and basal frontal regions and relative preservation of superior brain regions, the central region and the basal ganglia. Relative hyperaemia of the left hippocampus region was not statistically significant after correction for multiple comparisons, but contrasts with the expectation of lowered flow in basal temporal regions which are a major target of traumatic brain injury. ${ }^{810}$ Comparison with the control data revealed that the flow index of the patient group was very close to that obtained by controls when examined while imagining the visual appearance of faces. ${ }^{18}$ Likewise, in the right hippocampal region the numerical value of the patients' flow index was higher than the controls' flow index during rest, but nearly identical to that during visual imagery. Possibly, the elevation of the patients' hippocampal flow indices reflected engagement in mental activities during the time of isotope uptake.

Relationship of SPECT to neuropsychological performance and functional capacities

In brain damage the relationship of local blood flow to neuropsychological function is likely to be non-linear. There may be a lower margin of local blood flow beyond which regional function breaks down. Particularly if hypoperfusion is caused by tissue destruction, local flow may fall below that threshold, but the additional decrease cannot be accompanied by a corresponding decline of a function that has already been abolished at the threshold level. Therefore, non-parametric rank correlations were computed.

In a first step, 16 correlations to test scores were computed for each of the 34 regions. For each region up to 4.8 correlation coefficients with a P-value below 0.05 could be expected to result from mere chance (Binomial distribution, $\mathbf{P}=0.05)^{33}$. Five or more significant correlations occurred only for the right central region and for both thalami. The correlations of the central region were all negative, that is, a higher flow was associated with worse performance, whereas those of the thalami were positive.

Three steps were taken to enhance the power of correlations and to reduce data for making them amenable to interpretation. First, analyses of correlations were restricted to the thalamus and to the temporal and frontal regions which had previously been expected to be related to dysexecutive syndrome and memory. Second, larger regions were created by averaging across middle, inferior, anterior, and orbitofrontal regions, as well as across hippocampus, inferior and superior temporal regions. Third, the mean indices of frontal, temporal and thalamic regions were divided through the mean index of basal ganglia and central regions. The last step was based on the assumption that the elevations of flow indices of basal ganglia and central regions reflect relative sparing of these regions in the presence of diffuse hypoperfusion and therefore augment proportionally to the severity of diffuse hypoperfusion. The division then results in an index which diminishes as a sequel of both, local and diffuse hypoperfusion.

The resulting correlation coefficients are displayed in table 2 . The number of coefficients with a probability below 0.05 was above chance for the right frontal $(6 / 16)$ and both thalamic regions (left: $5 / 16$; right: $8 / 16$ ) and close to the upper chance limit for the left frontal region (4/16). It did not exceed chance for both temporal regions (left: $2 / 16$; right: 1/16), and one of both significant correlations of the left temporal region associated higher flow values with worse test performance. Both of the remaining positive correlations of the temporal lobes concerned the vocabulary test.

Three of 4 correlations of frontal and thalamic regions to functional capacities, and 7 out of 8 to intelligence tests had a probability below 0.05 . The proportion of significant relationships was lower but reliably above chance for executive functions (14/34), and at chance level for tests of secondary memory (1/16).

Since there was neither a significant number of correlations between temporal flow values and any test scores nor between memory tests 
and any regional flow indices, the respective correlation coefficients will not be further analysed.

Out of the remaining 48 correlations only those of the right thalamus to design fluency, card sorting, vocabulary and progressive matrices, and that of the left thalamus to vocabulary were reliable at a global alpha level of 0.05 after Bonferroni-Holms correction. ${ }^{32}$ Our interpretation will concentrate upon the pattern of relationships rather than the significance of single correlation coefficients.

To assess the general level of the strength of correlations of frontal and thalamic regions to executive functions, intelligence and functional capacities, their mean values were computed. They were $0 \cdot 19$ (SD $0 \cdot 14$ ) for the left frontal, 0.28 (SD 0.19) for the right frontal, 0.26 (SD $0 \cdot 16$ ) for the left thalamus, and 0.35 (SD 0.17) for the right thalamus. All of these values differ significantly from zero ( $t$ test, $\mathrm{p}<0.05$ for left frontal region and $p<0.01$ for the remainder). Correlations of right brain regions were stronger than those of homologous left regions for both the frontal region and thalamus, but the differences did not reach statistical significance ( $t$ test, $\mathrm{p}>0.05$ ). Correlations to the thalami were also numerically stronger than those of ipsilateral frontal regions, but the differences fell short of any statistical significance.

Because correlations to memory tests did not differ from zero, an analysis of differences between correlations to verbal and visuospatial memory tests was not justified. For word fluency correlations to left brain regions were stronger than those to right brain regions whereas the reverse was the case for design fluency, but for both tests the differences between the correlation-coefficients to corresponding right and left brain regions were far from statistical significance. Both verbal and spatial working memory correlated stronger with right than with left brain regions.

\section{Discussion}

One purpose of the study was to explore the effects of frontal and temporal lobe dysfunction on executive and memory function in SHI. On this question the results are disappointing. Tests of secondary memory did not significantly correlate with any flow values, and temporal lobe flow values did not significantly correlate with any psychological test except for vocabulary. Frontal flow values did show significant correlations to executive function, but somewhat stronger relationships were found with the thalamic regions which had not been considered in the dichotomy of frontal and temporal brain regions.

In interpreting the absence of correlations between memory tests and flow values it should be kept in mind that SHI patients scored worse than controls on all of the memory tests. This indicates that the tests were sensitive to the effect of SHI. Possibly the relevant lesions were not captured by SPECT. This might easily be the case if they were located in the hippocampus region which occupies only a small band at the mesial border of the temporal lobe. Although the region is a main target of various mechanisms of traumatic brain injury ${ }^{810}$ its flow rates in the patient group were not lower than those of controls. The calculation of a compound index across all temporal regions further attenuated the impact of hippocampal flow variations on the results of the correlational analysis, but this step was taken only after it had been found that the hippocampal regions alone did not significantly correlate with any test results. The comparison with the complete SPECT data from the control study ${ }^{18}$ suggested that hippocampal flow rates in patients were subject to functional variations caused by spontaneous mental activities during SPECT. This additional source of variation may have contributed to a masking of relationships between the basic flow rates of the region and memory capacity.

There are three main ways to account for the relationship between thalamic flow values and neuropsychological performance in SHI. All start from the observation that the thalamus has rich reciprocal connections with many cortical areas. One possibility is that damage to the thalamus caused deafferentiation and hence dysfunction of extended cortical areas. Primary lesions in the thalamus can result from rupture of small perforating vessels. ${ }^{8}$ They have been documented on post-mortem ${ }^{8}$ and $\mathrm{NMR}^{910}$ in patients with very severe head injury and DAI. The second possibility is that cortical brain damage caused dysfunction of connected thalamic nuclei which manifested itself in a reduction of thalamic flow indices. ${ }^{34}$ Because afferents from widespread and diverse cortical areas converge on the thalamus, effects of multiple and heterogeneous cortical lesions may sum up to make thalamic flow an indicator of the total amount rather than of the distinct location of cortical lesions. The third possibility is, that its functional dependence on connections with the cortex makes thalamic function particularly vulnerable to the effects of multiple disconnections caused by DAI. Strich ${ }^{15}$ found degeneration of thalamic nuclei in all of their patients with severe DAI who had survived the trauma for several months. She thought that those nuclei were affected whose afferent or efferent connections had been interrupted and that the thalamus lesions were secondary to the degeneration of the white matter. Adams ${ }^{7}$ confirmed in a larger series of patients that there is a loss of intrinsic architecture and a reduction in size of the thalami in DAI. If reduction of thalamic blood flow was caused by dysfunction or degeneration secondary to DAI, its association to neuropsychology could be an indication of how strongly psychological functions are affected by DAI rather than indicating the impact of thalamic damage per se on these functions.

Although several tests had explicitly been selected to be differentially sensitive to verbal and visuospatial dysfunction and hence to left and right brain damage, laterality of flowreduction had little if any differential influence on their results. Generally, flow in right brain regions appeared to have somewhat stronger 
effects upon test performance and functional capacities than that of corresponding left brain regions, although none of the differences reached statistical significance. Apart from their effect on cognitive functions, right hemisphere lesions may lead to emotional disturbances. Patients with right hemisphere lesions have a higher incidence of anosognosia $^{35}$ and frequently tend to disregard adverse sequels of their disease. ${ }^{36}$ Right brain damage can also lead to disregard or misperception of emotional and pragmatic implications of communication. ${ }^{37-40}$ Possibly, these emotional and communicative disturbances affect not only functional capacities in daily living ${ }^{13-541}$ but also the patient's success on neuropsychological tests which are not specifically sensitive to the cognitive sequelae of right brain damage.

The results of this study were presented at the symposium on Functional neuroimaging and neuropsychology in diffuse brain damage at the 13th European INS Conference, Innsbruck, 4-7 July, 1990.

Footnote 1

Compound Score $=$ Score on 2 nd run $-10^{\star}$ (Difference from first to second run / Score on first run). The upper limit was set to 30 , that is to the maximum score of one run, so that an increase from the first to the second run could not elevate the compound score above the score achieved by perfect performance on both runs.

Footnote 2

Compound Score $=$ Score on 2 nd run $-3^{\star}$ (Difference from first to second run / Score on first run).

1 Thomsen IV. Late outcome of severe blunt head trauma: a 10-15 year second follow-up. 7 Neurol Neurosurg Psychiatry 1984;47:260-8.

2 Van Zomeren AH, Van Den Burg W. Residual complaints of patients two years after severe head injury. 7 Neurol Neurosurg Psychiatry 1985;48:21-8.

3 McKinlay WW, Brooks DN, Bond MR, Martinage DP, Marshall MM. The short-term outcome of severe blunt head injury as reported by relatives of the injured person. head injury as reported by relatives of the injured

4 Brooks N. Behavioural and social consequences of severe head injury. Traumatic brain injury-clinical, social and rehabilitational aspects. In: Deelman BG, Saan RJ, van Zomeren AH, eds. Amsterdam/Lisse: Swets and Zeitlinger, 1990:77-88.

5 Brooks N, Campsie L, Symington C, Beattie A, McKinlay $W$. The five year outcome of severe blunt head injury: a relative's view. $\mathcal{f}$ Neurol Neurosurg Psychiatry 1986; 49:746-70.

6 Mattson AJ, Levin HS. Frontal lobe dysfunction following closed head injury - a review of the literature. 7 Nerv Ment Dis 1990;178:282-91.

7 Adams JH. The neuropathology of head injuries. In: Vinken PJ, Bruyn GW, eds. Handbook of clinical neurology. Amsterdam: North Holland Publishing, 1975:35-65.

8 Adams JH, Graham DI, Scott G, Parker LS, Doyle D. Brain damage in fatal non-missile head injury. $\mathcal{f}$ Clin Pathol 1980;33:1132-45.

9 Jenkins A, Teasdale G, Hadley MDM, Macpherson P, Rowan JO. Brain lesions detected by magnetic resonance imaging in mild and severe head injuries. Lancet imaging in mild

10 Gentry LR, Godersky JC, Thompson B. MR imaging of head trauma: Review of the distribution and radiopathologic features of traumatic lesions. Amer $\mathcal{f}$ Neuroradiol 1987;9:101-10.

11 Levin HS, Amparo E, Eisenberg HE, et al. Magnetic resonance imaging and computerised tomograph in relation to the neurobehavioural sequelae of mild to moderate head injuries. $\mathcal{F}$ Neurosurg 1987;66:706-13. 12 Levin HS, Williams D, Crofford MJ, et al. Relationship of closed head injury. $\mathcal{F}$ Neurosurg 1988;69:861-6.
13 Wilson JTL, Wiedmann KD, Hadley DM, Condon B, Teasdale G. Early and late magnetic resonance imaging and neuropsychological outcome after head injury. 7 Neurol Neurosurg Psychiatry 1988;51:391-6.

14 Wilson JTL Wiedmann KD, Hadley DM, Brooks DN. The relationship between visual memory function and lesions detected by magnetic resonance imaging after closed head injury. Neuropsychology 1989;3:255-66.

15 Strich SJ. Diffuse degeneration of the cerebral white matter in severe dementia following head injury. $\mathcal{f}$ Neurol Neurosurg Psychiatry 1956;19:163-85.

16 Wiedmann KD, Wilson JTL, Wyper D, Hadley DM Teasdale GM, Brooks DN. SPECT cerebral blood flow, MR imaging, and neuropsychological findings in traumatic head injury. Neuropsychology 1989;3:267-81.

17 Uzzell BP. Pathophysiology and behavioural recovery. In: Uzzell BP, Gross Y, eds. Clinical neuropsychology of intervention. Dordrecht: Martinus Nijhoff, 1986:3-18.

18 Goldenberg G, Podreka I, Uhl F, Steiner M, Willmes K Deecke L. Cerebral correlates of imagining colours, faces and a map. I. SPECT of regional

19 Goldenberg G, Podreka I, Mueller C, Deecke L. The relationship between cognitive deficits and frontal lobe function in patients with Parkinson's disease: An emission computerised tomography study. Behav Neurol 1989;2. 79-87.

20 Shallice T. Specific impairments of planning. Phil Trans $R$ Soc Lond B 1982;298:199-209.

21 Regard M, Straquss E, Knapp P. Children's production on verbal and non-verbal fluency tasks. Percept Mot Skills 1982;55:839-44.

22 Daneman $M$, Carpenter PA. Individual differences in working memory and reading. F Verb Learn Verb Behav 1980;19:450-66.

23 Goldenberg G. Performance of concurrent non-motor tasks in Parkinson's disease. $₹$ Neurol 1990;237:191-6.

24 Baddeley A. Working memory. Oxford: Oxford University Press, 1986.

25 Lezak MD. Neuropsychological assessment. Oxford: Oxford University Press, 1983

26 Lehrl S. Mehrfachwahl-Wortschatz-Intelligenztest. MWT-B Erlangen: Verlag D Straube, 1977.

27 Raven JC. Coloured progressive matrices. HK Lewis: London, 1965.

28 Shoulson I. Huntington's disease: functional capacities in patients treated with neuroleptic and antidepressan drugs. Neurology 1981;31:1333-5.

29 Podreka I, Suess E, Goldenberg G, et al. Initial experience with Tc-99m-Hexamethylpropyleneamineoxime (Tc99m-HM-PAO) brain SPECT. f Nucl Med 1987; 28:1657-66.

30 Lassen NA, Andersen AR, Friberg H, Neirinckx RD. Technetium-99m-HMPAO as a tracer of cerebral blood fechnetium-99m-HMPAO as a tracer of cerebral blood Metab 1987;7(S):535.

31 Heiss WD, Herholz K, Podreka I, Neubauer I, Pietrzyk U. Comparison of $99 \mathrm{~m}$-Tc-HMPAO SPECT with $18-\mathrm{F}$ Fluoromethane PET in cerebrovascular disease. $f$ Cereb Blood Flow Metab 1990;10:687-97.

32 Holms S. A simple sequentially rejective multiple test procedure. Scand $\mathcal{F}$ Statist 1979;6:65-71.

33 Diem K, Lentner C. "Wissenschaftiche Tabellen". CibaGeigy; Basel 1968.

34 Metter EJ, Wasterlein CG, Kuhl DE, Hanson WR, Phelps ME. 18-FDG-Positron Emission Computed Tomography in a study of aphasia. Ann Neurol 1981;10:173-83.

35 Cutting J. Study of anosognosia. F Neurol Neurosurg Psychiatry 1978;41:548-55.

36 Gainotti G. Emotional behaviour and hemispheric side of the lesion. Cortex 1972;8:41-55.

37 Heilman KM, Schwartz HD, Watson RT. Hypoarousal in patients with the neglect syndrome and emotional indifpatients with the neglect syndrome

38 Brownell HH, Michel D, Powelson J, Gardner H. Surprise but not coherence: Sensitivity to verbal humour in righthemisphere patients. Brain Lang 1983;18:20-7.

39 Hecaen $H$. Right hemisphere contribution to language functions. In: Buser P, Rougeul-Buser A, ed. Cerebral correlates of conscious experience. Amsterdam: Elsevier, 1978:119-214.

40 Ostrove JM, Simpson T, Gardner H. Beyond scripts: A note on the capacity of right hemisphere-damaged patients to process social and emotional content. Brain Cogn 1990;12:144-54.

41 Prigatano GP. Emotion and motivation in recovery and adaptation after brain damage. In: Finger S, LeVere TE, Almli CR, Stein DG, eds. Brain injury and recoverytheoretical and controversial issues. NewYork: Plenum Press, 1988:335-50. 